\title{
Lupoid cutaneous leishmaniasis due to Leishmania major, a great mimicker of Lupus Vulgaris in Sanliurfa, Turkey: a case report
}

\author{
Nebiye Yentur Doni ${ }^{1}$, Gulcan Gurses ${ }^{2}$, and Isa $\mathrm{An}^{3}$ \\ ${ }^{1}$ Harran University Faculty of Medicine \\ ${ }^{2}$ Harran University Vocational School of Health Services, Department of Microbiology and \\ Parasitology \\ ${ }^{3}$ Sanlıurfa Training and Research Hospital
}

May 18, 2020

\begin{abstract}
Lupoid cutaneous leishmaniasis is identified by a broad range of clinical presentation with brown to red, yellowish-brown, applejelly papules. These papules enlarge to plaques, develop into granulomatous, crusted ulcers, which are as similar as cutaneous lesions of lupus vulgaris. Herein, we report a patient with lupoid CL mimicking lupus vulgaris
\end{abstract}

\section{Lupoid cutaneous leishmaniasis due toLeishmania major, a great mimicker of Lupus Vulgaris in Sanliurfa, Turkey: a case report INTRODUCTION}

Cutaneous leishmaniasis (CL) is a dermal infection caused mostly by Leishmania major, Leishmania tropica , and Leishmania infantum, is transmitted to humans by the bite of sandflies. Cutaneous leishmaniasis is polymorphic and may result in lesions characterized by different clinical features. Cutaneous leishmaniasis is also characterized by acute or chronic course. Lupoid cutaneous leishmaniasis (LCL) is a clinical form of CL characterized by a chronic relapsing course, unusual clinical features, an unique granulomatous lesion ${ }^{1}$. Lupoid cutaneous leishmaniasis is identified by a broad range of clinical presentation with brown to red papules, yellowish-brown papules and, apple-jelly papules. These papules enlarge to plaques, and develop into granulomatous and crusted ulcers, which are as similar as cutaneous lesions of lupus vulgaris. Herein, we report a patient with lupoid CL mimicking lupus vulgaris to highlight the unusual clinical spectrum of CL.

Key words: Lupoid cutaneous leishmaniasis, lupus vulgaris,Leishmania major, Turkey

\section{Key Clinical Message}

Many unusual lesions might be caused by Leishmania.

\section{Case Report}

\section{Clinical Findings}

A 24 year old man presented presented with a 3-5 months history of a well-defined erythematous plaque extended to involve the whole left hand middle finger, resembling lupus vulgaris. He also presented 1 ulcerated lesion on his left shoulder, and 1 papulonodular lesion on his left arm. He was a textile worker. 
He could not use his left hand due to the huge lesion on his middle finger. While, his general physical examination and systemic examination were normal.

The patient had no familial history for the development of CL. He had no travel to other CL endemic provinces. The patient had applied to different medical clinics in non-endemic provinces for CL for the diagnosis of the lesions he had. He was diagnosed as "lupus vulgaris" but he had not been treated for lupus vulgaris or CL. Then the patient applied to our dermatological clinic in Sanliurfa, a hyper-endemic province for CL.

\section{Histopathological Findings}

Direct smears from the exudate of fluid of the lupoid lesion were stained with special stains Gram, ErlichZiehl-Nielsen (EZN), Periodic Acid-Schiff (PAS), Giemsa for detection of bacteria, mycobacteria, fungi and leishmania amastigotes, respectively. A punch biopsy aspiration fluid from lesions was used, and a part of the exudate of fluid of the lupoid lesion was used for the identification ofLeishmania species by Real Time PCR. Application of real time PCR that targeted the ITS1 region of Leishmania species using the DNAs obtained from the lesion of the patient. In this real time PCR, melting temperature of $L$. major reference control and sample is $85^{\circ} \mathrm{C}$ as described previously ${ }^{2}$.

\section{Results}

Written informed consent to enroll the study and use clinical images in case report was obtained from the patient.

Granuloma structures characterized by epithelioid histiocytes in the dermis were observed in the prepared sections. Langerhans-type multinucleate giant cells were noteworthy which were found in granulomas suggestive of leishmaniasis, tuberculosis with Hematoxylin-eosin staining (200X) (Figure 1). Intense mononuclear inflammatory cells surrounding the granuloma infiltrate the granuloma were also noteworthy. Granulomas were mostly observed in the dermis. The histomorphological findings seemed to be compatible with lupus vulgaris. However, a definitive differential diagnosis could not be performed.

No bacteria was observed with Gram staining, no Mycobacteria was observed with EZN staining and no fungal organism was observed with PAS staining.

A few amastigotes of Leishmania were observed with Giemsa staining. Detection of Leishmania amastigotes by the microscopic examination of the direct smears exudative fluid of the lesions strictly supported the definitive differrantial diagnosis of lupoid CL. It has been reported that Leishmania amastigotes are usually absent on microscopy, thus, PCR is especially recommended for diagnosis of CL to prevent the delayed diagnosis or clinical and histological misdiagnosis often with Lupus vulgaris ${ }^{3}$. In light of this data, Real Time PCR was used to identify the Leishmaniaspecies, which was found to be L. major (Figure 2).

Figure 1. Non-caseified granulomas including giant cells (green arrow) and Leishmaniaamastigotes (red arrow) were seen in dermis (Hematoxylin-eosin, X200)

Figure 2. Real-time PCR analysis of Leishmania major infection in lesion of the patient

Identification of polymerase chain reaction products with melting temperature analysis of two L. major samples and two samples ofLeishmania major belonged to the patient. The melting temperature is $85^{0} \mathrm{C}$ for L. major MHOM/SR/2015/HRURFA012.

The patient with lupoid CL (Figure 3) was treated with intralesional meglumine antimoniate (Glucantime@) injections twice a week (Monday-Thursday) for four weeks. After four weeks, the patient was called monthly for control the healing of the lesion for one year, it was seen that the lesion was regressed (Figure 4).

The plaque type occurred over the finger and presented clinically as well-defined erythematous plaques that sometimes extended to involve the whole finger resembling lupus vulgaris.

Figure 3. Lesion of the lupoid CL before treatment 
Figure 4. Lupoid CL, after treatment of the lesion with intralesional meglumine antimoniate (Glucantime@)

\section{Discussion}

Leishmaniasis is a neglected vector-borne disease caused by protozoan parasites belonging

to Leishmania genus, and endemic in all WHO regions, including Turkey. There are 4 main clinical classifications of the leishmaniasis, visceral, post-kala-azar dermal, cutaneous (CL) and mucocutaneous. Turkey is considered to have a high burden country for $\mathrm{CL}^{4}$. Although a National control programme has been established, CL is still a serious public health problem with increasing the number of CL cases due to the Syrian conflict in Turkey, particularly in Southeastern and Southern regions of the country. Sanliurfa is a hyper-endemic province of CL in Southeastern region of Turkey. Various clinical presentations of CL including papule, nodule, ulcerative, noduloulcerative, and recidivans forms have been reported in Sanliurfa ${ }^{5-8}$. Noduloulcerative form, classically known as oriental sore, is the most common form in study area.

To the best of our knowledge, lupoid CL mimicking lupus vulgaris has not been reported from Sanliurfa, Southeastern Region of Turkey. However, a few lupoid CL cases have been reported in different provinces of Turkey ${ }^{9 ; 10}$. Of all CL cases the incidence of Leishmaniasis recidivans has been reported with $0,5 \%-6,2 \%$ in the Middle East and Afghanistan ${ }^{11}$. In a recent study carried out in Tunisia, lupoid CL represented $9 \%$ of cases in adults ${ }^{12}$. It has been reported that in lupoid CL, theLeishmania amastigotes are usually absent or rare on a microbiological smear ${ }^{13-15}$. The paucity ofLeishmania amastigotes in the lesion samples and in direct smears might lead to misdiagnosis with lupus vulgaris ${ }^{11 ; 13-15}$. Since, it has been reported that clinically and histologically, lupoid leishmaniasis is similar to lupus vulgaris, which is considered as the most important differential diagnosis ${ }^{16 ; 17}$. Contrary to other studies ${ }^{13-15}$, we detected fortunately Leishmania amastigotes in direct smears of the lesion of the patient. This can be explained by the short duration of the patient's lesions (approximately 3-5 months) at the moment of diagnosis. We detected also Leishmania DNA by ITS 1 PCR and identified by Real time PCR. We found that L. major is responsible agent for this LCL case, which is compatible with the other studies ${ }^{14 ; 18 ; 19}$. Leishmania tropica is considered to be the most common, and L. major is the rare causative agent of lupoid $\mathrm{CL}^{11 ; 14 ; 15 ; 20}$. In a study it has been reported species-specific PCR analysis is sensitive in cases of acute cutaneous leishmaniasis, but in lupoid leishmaniasis it is less sensitive ${ }^{14}$.

There is no standardized treatment for this condition and thus multiple treatments have been reported with varying degrees of success. Treatment options include cryotherapy, topical antimonial compounds and intralesional pentavalent antimony. This patient was treated with intralesional meglumine antimoniate (Glucantime $\mathbb{B}$ ) injections twice a week for four weeks with marked improvement of clinical features.

\section{Conclusion}

Lupoid CL can be manifested as an uncommon form that can mimic many other skin disorders as lupus vulgaris. Hence, the physicians living in nonendemic areas should never overlook that many unusual lesions might be caused by Leishmania. Any ignoring may be resulted in missed and delay the precise diagnosis, submitting the patients to inappropriate prescribing of drugs, increased resistance to treatment, worsening the clinical presentation, and contributing to the transmission chain of the parasite. Correct and reliable identification of parasite might contribute for breaking the chain of transmission of the parasite and decrease the prevalence. Diagnosis of CL should be performed in all patients from endemic areas of Leishmaniasis by both microscopic examination of Giemsa stained smears and PCR and identified of Leishmania species by using a PCR-RFLP technique.

\section{Author Contribution}

Conceptualization: Nebiye Yentur Doni, Gulcan Gurses, İsa An.

Data curation: Nebiye Yentur Doni, Gulcan Gurses.

Formal analysis: Nebiye Yentur Doni. 
Funding acquisition: Nebiye Yentur Doni.

Investigation: Nebiye Yentur Doni, Gulcan Gurses.

Methodology: Nebiye Yentur Doni, Gulcan Gurses, İsa An.

Project administration: Nebiye Yentur Doni

Resources: Nebiye Yentur Doni.

Software: Nebiye Yentur Doni, Gulcan Gurses, İsa An.

Supervision: Nebiye Yentur Doni, Gulcan Gurses

Validation: Nebiye Yentur Doni, Gulcan Gurses, İsa An.

Visualization: Nebiye Yentur Doni, Gulcan Gurses.

Writing \pm original draft: Nebiye Yentur Doni.

Writing \pm review \& editing Nebiye Yentur Doni, Gulcan Gurses, İsa An.

\section{References}

1 R. B. Mlika, H. Hammami, A. Sioud, I. Mokhtar, S. Fenniche. THERAPEUTIC HOTLINE Lupoid leishmaniasis of the nose responding well to cryotherapy. Dermatologic Therapy 2011; 24: 378-379.

2 B. O. Owino, D. Matoke-Muhia, Y. Alraey et al. Association of Phlebotomus guggisbergi with Leishmania major and Leishmania tropica in a complex transmission setting for cutaneous leishmaniasis in Gilgil, Nakuru county, Kenya. PLoS Negl Trop Dis 2019; 13: e0007712.

3 WHO. World Health Organization. Report of a meeting of theWHO Expert Committee on the Control of Leishmaniases, Geneva. WHO Tech Series2010: 23-129.

4 WHO. Weekly Epidemiological Record, Global leishmaniasis surveillance update, 1998-2016. World Health Organization 2018; 93: 521-540.

5 M. Aksoy, N. Doni, H. U. Ozkul et al. Pediatric Cutaneous Leishmaniasis in an Endemic Region in Turkey: A Retrospective Analysis of 8786 Cases during 1998-2014. PLOS Neglected Tropical Diseases2016; 10: e0004835.

6 G. Gurses, M. Ozaslan, F. Y. Zeyrek et al. Molecular identification of Leishmania spp. isolates causes cutaneous leishmaniasis (CL) in Sanliurfa Province, Turkey, where CL is highly endemic. Folia Microbiol (Praha) 2018; 63: 353-359.

7 E. Turan, Y. Ye ilova, H. A. Surucu et al. A Comparison of Demographic and Clinical Characteristics of Syrian and Turkish Patients with Cutaneous Leishmaniasis. Am J Trop Med Hyg 2015; 93: 559-563.

8 N. Yentur Doni, G. Gurses, R. Dikme et al. [Investigation of cutaneous leishmaniasis by active screening in primary schools in Sanliurfa, Turkey]. Mikrobiyol Bul 2016; 50: 559-568.

9 K. Cingöz, A. Türel Ermertcan, P. Temiz. Cutaneous leishmaniasis mimicking lupus vulgaris. DOD Clinical Case Report 2018; 2: 23-26.

10 A. Ferahbas, S. Mistik, S. Utas et al. Cutaneous lupoid leishmaniasis: A case report. Cutis 2006; 77: $25-28$.

11 M. S. Gurel, M. Ulukanligil, H. Ozbilge. Cutaneous leishmaniasis in Sanliurfa: epidemiologic and clinical features of the last four years (1997-2000). Int J Dermatol 2002; 41: 32-37.

12 I. Zaraa, F. Ishak, R. Kort et al. Childhood and adult cutaneous leishmaniasis in Tunisia. Int J Dermatol 2010; 49: 790-793. 
13 A. Masmoudi, S. Boudaya, N. Ayadi et al. Clinical and histological study of lupoid cutaneous leishmaniasis (16 cases).Presse Medicale 2007; 36: 1738-1742.

14 A. Z. Momeni. Chronic Lupoid Leishmaniasis. Archives of Dermatology 1996; 132: 198.

15 A. Ul Bari, N. Raza. Lupoid cutaneous leishmaniasis: A report of 16 cases. Indian Journal of Dermatology Venereology \& Leprology2010; 76.

16 M. A. Nilforoushudeh, G. Sadeghian, F. Jaffary, H. Ziaei, L. Shirani-Bidabad, P. Mahzoni. Successful treatment of lupoid cutaneous leishmaniasis with glucantime and topical trichloroacetic acid (a case report). Korean Journal of Parasitology 2008; 46: 175-177.

17 A. Herrmann, J. Wohlrab, H. Sudeck, G. D. Burchard, W. C. Marsch. [Chronic lupoid leishmaniasis. A rare differential diagnosis in Germany for erythematous infiltrative facial plaques]. Hautarzt2007; 58: 256-260.

18 A. Khaled, S. Goucha, S. Trabelsi, R. Zermani, B. Fazaa. Lupoid cutaneous leishmaniasis: a case report. Dermatol Ther (Heidelb)2011; 1: 36-41.

19 G. Sadeghian, H. Ziaei, L. Shirani-Bidabadi, M. A. Nilforoushzadeh. Lupoid leishmaniasis due to Leishmania major with remaining large scars: report of 2 cases. Eastern Mediterranean Health Journal 2010; 16: 344-345.

20 T. Narang, S. Dogra, A. J. Kanwar, B. D. Radotra. Disfiguring ulcerated plaques - Lupoid leishmaniasis. Clinical and Experimental Dermatology 2008; 33: 805-807.

\section{Hosted file}

figure_1.docx available at https://authorea.com/users/322578/articles/451536-lupoidcutaneous-leishmaniasis-due-to-leishmania-major-a-great-mimicker-of-lupus-vulgarisin-sanliurfa-turkey-a-case-report

\section{Hosted file}

figure_2.docx available at https://authorea.com/users/322578/articles/451536-1upoidcutaneous-leishmaniasis-due-to-leishmania-major-a-great-mimicker-of-lupus-vulgarisin-sanliurfa-turkey-a-case-report

\section{Hosted file}

figure_3.docx available at https://authorea.com/users/322578/articles/451536-1upoidcutaneous-leishmaniasis-due-to-leishmania-major-a-great-mimicker-of-lupus-vulgarisin-sanliurfa-turkey-a-case-report

\section{Hosted file}

figure_4.docx available at https://authorea.com/users/322578/articles/451536-lupoidcutaneous-leishmaniasis-due-to-leishmania-major-a-great-mimicker-of-lupus-vulgarisin-sanliurfa-turkey-a-case-report 